\title{
Optimal diagnosis and disease activity monitoring of intestinal Behçet's disease
}

\author{
Hyun Jung Lee*, Jae Hee Cheon* \\ Department of Internal Medicine and Institute of Gastroenterology, Yonsei University College of Medicine, Seoul, Korea
}

Intestinal Behçet's disease (BD), generally accepted as a type of inflammatory bowel disease (IBD), could be diagnosed when patients with BD have objectively documented gastrointestinal symptoms and intestinal ulcerations. Similar to IBD, intestinal $\mathrm{BD}$ has an unpredictable disease course with relapse and remission and is often related to a poor prognosis. However, there is no single gold standard for assessment of the disease activity of intestinal BD, and its diagnosis and management depend heavily on expert opinions. The Korean IBD Study Group recently developed novel diagnostic criteria based on colonoscopy findings and clinical manifestations using a modified Delphi process to overcome drawbacks of previously used consensus for the diagnosis of intestinal BD. In addition, the recently developed disease activity index for intestinal BD, consisting of a relatively simple 8-point index, could also help in determining treatment strategies and monitoring therapeutic responses. In this review, the progress in the diagnosis and disease activity measurement of intestinal BD will be discussed. (Intest Res 2017;15:311-317)

Key Words: Behcet Syndrome; Inflammatory bowel disease; Diagnostic criteria; Disease activity index

\section{INTRODUCTION}

Behçet's disease (BD) is a multisystemic, chronic, relapsing systemic inflammatory disorder characterized mainly by oral and genital ulcers, uveitis, skin manifestations, and joint, vascular, CNS, and gastrointestinal involvements. ${ }^{1-3}$ When patients with BD present with predominant gastrointestinal symptoms and intestinal ulcerations observed objectively, they may be diagnosed with intestinal $\mathrm{BD}^{4,5}$ The prevalence of intestinal BD is higher in East Asia, including Korea and Japan, than in Mediterranean countries. ${ }^{6}$ Similar to IBD, in- testinal BD shows a fluctuating clinical course with repeated episodes of relapses and remissions. ${ }^{7,8}$ Gastrointestinal involvement of $\mathrm{BD}$, often related to poor treatment responses and catastrophic bowel complications, can be life-threatening in some cases, ${ }^{9,10}$ although symptoms of intestinal BD range from mild abdominal discomfort to massive bleeding, fistula, or bowel perforation. ${ }^{6,7}$ Indeed, intestinal BD requires similar surgical interventions to those of $\mathrm{CD}$, predicting a poor prognosis. ${ }^{11}$ However, clinical data regarding the diagnosis of intestinal $\mathrm{BD}$ and predicting factors for its disease activity and long-term outcomes are still limited because of

Received December 27, 2016. Revised January 20, 2017. Accepted January 23, 2017. Published online April 25, 2017

Correspondence to Jae Hee Cheon, Department of Internal Medicine, Yonsei University College of Medicine, 50- 1 Yonsei-ro, Seodaemun-gu, Seoul 03722, Korea.

Tel: +82-2-2228-1990, Fax: +82-2-393-6884, E-mail: geniushee@yuhs.ac

Co-Correspondence to Hyun Jung Lee, Department of Internal Medicine, Yonsei University College of Medicine, 50-1 Yonsei-ro, Seodaemun-gu, Seoul 03722,

Korea. Tel: +82-2-2228-1989, Fax:+82-2-393-6884,E-mail: guswjd@yuhs.ac

*This paper has two corresponding authors.

*These authors contributed equally to this study.

Financial support: This research was supported by a grant (HI13C1345) from the Korean Health Technology RetD Project through the Korea Health Industry Development Institute (KHIDI), which is funded by the Ministry of Health and Welfare, Republic of Korea; a grant (NRF-2013R1A2A2A01067123) from the Basic Science Research Program through the National Research Foundation of Korea, which is funded by the Ministry of Science, ICT and Future Planning; and a faculty research grant (2012-31-0477) from the Department of Internal Medicine, Yonsei University College of Medicine. Conflict of interest: None.

\footnotetext{
(c) Copyright 2017. Korean Association for the Study of Intestinal Diseases. All rights reserved.
}

This is an Open Access article distributed under the terms of the Creative Commons Attribution Non-Commercial License (http://creativecommons.org/licenses/by-nc/4.0)

which permits unrestricted non-commercial use, distribution, and reproduction in any medium, provided the original work is properly cited. 
its scarcity. The Korean IBD Study Group recently proposed novel diagnostic criteria and an index to assess the disease activity of intestinal BD. Here, we will review the progress in the diagnosis and assessment of the disease activity of intestinal BD.

\section{DIAGNOSIS OF INTESTINAL BD}

The incidence of gastrointestinal involvement in BD shows a remarkable geographic variation, ranging from $0 \%$ to $60 \%$, which seems to be more prevalent in East Asia. ${ }^{6,12}$ Although the gastrointestinal involvement of BD may be related to a poor prognosis in affected patients, to date, no specific diagnostic criteria have been developed for intestinal $\mathrm{BD}$ because of its rareness and lack of clinical evidence.

Intestinal BD is generally diagnosed if patients have both intestinal ulcerations and clinical findings of systemic BD. Diagnosis should be made considering the clinical, endoscopic, radiologic, and pathologic findings. ${ }^{13,14}$ The clinical manifestations need to meet the diagnostic criteria of BD as suggested by the International Study Group for BD or the BD Research Committee of Japan. ${ }^{15,16}$ The common gastrointestinal symptoms include abdominal pain, diarrhea, bleeding, and weight loss, and the typical intestinal ulcerations are defined as $<5$ ulcers that are oval in shape and deep with discrete borders located in the ileocecal area (Fig. 1). ${ }^{17}$ However, various ulcerations could be observed from aphthous ulcers to deep and penetrating volcano-shaped ulcers, and not only focal distribution but also diffuse ulcerations present in patients with intestinal $\mathrm{BD} .^{18}$ The representative pathologic findings of intestinal BD include the presence of vasculitis involving small- and medium-sized vessels and lymphocyte infiltration in the perivascular space. ${ }^{19,20}$ The absence of a noncaseating granuloma would indicate intestinal BD rather than CD, although such a finding is identified in less than half of the CD cases.

\section{Differential Diagnosis}

Other diseases that mimic intestinal BD should be ruled out before intestinal BD is diagnosed, including tuberculosis, CD, nonspecific colitis, and drug-associated colitis. ${ }^{21}$ Intestinal BD and CD indeed share numerous similarities, such as nonspecific gastrointestinal symptoms, extraintestinal manifestations, wax-and-wane disease courses, and long-term clinical outcomes; thus, it is sometimes difficult to distinguish between the $2{ }^{7,11}$ However, clinically, oral and genital ulcerations are more common in intestinal $\mathrm{BD}$, while
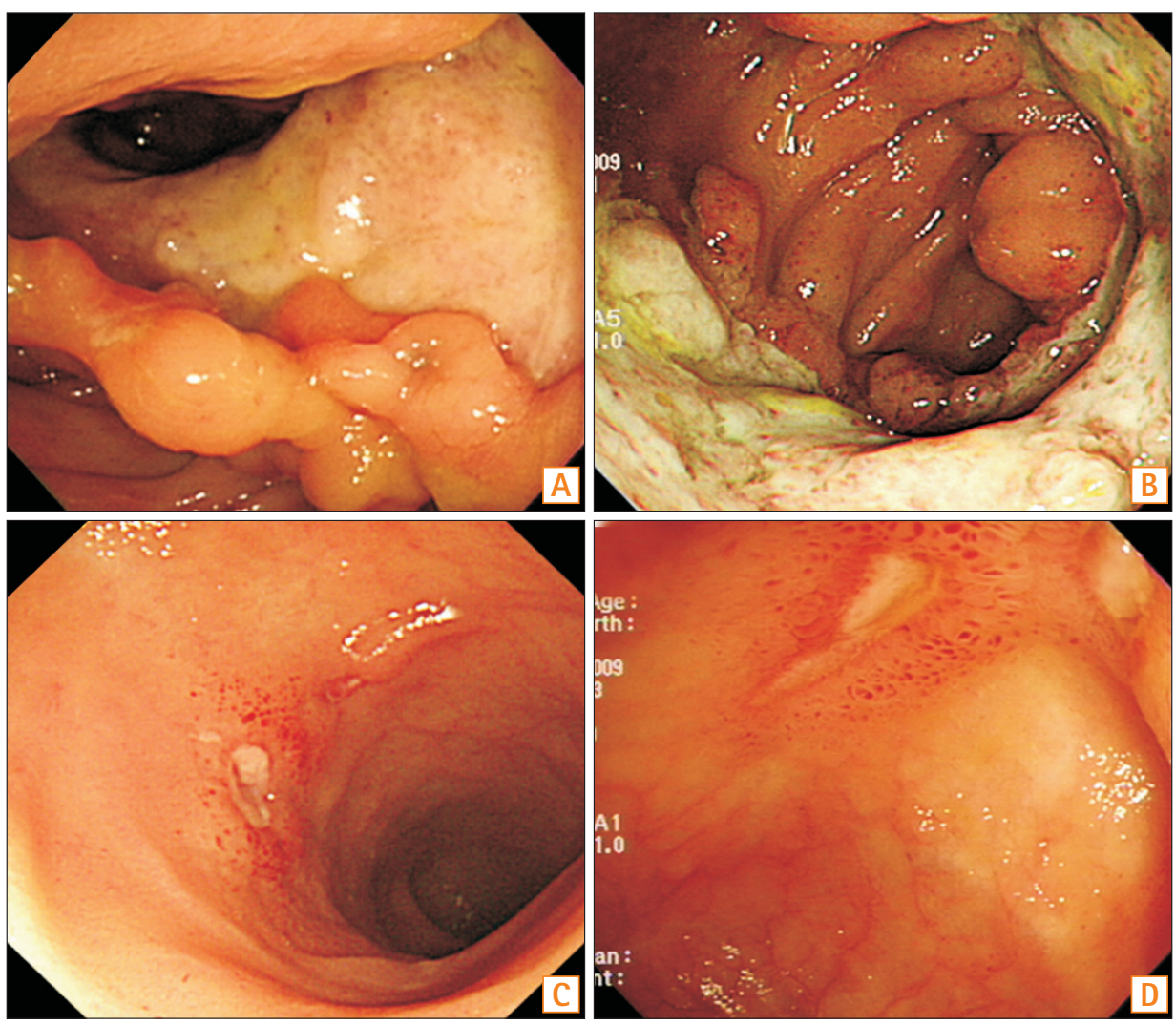

Fig. 1. Colonoscopic findings of intestinal Behçet's disease. (A, B) Typical ulcers for intestinal Behçet's disease are defined as single or few deep oval-shaped ulcers with discrete margins in the ileocecal area. (C, D) Atypical ulcers for intestinal Behçet's disease are defined as intestinal ulcerations that do not meet all of these characteristics, such as aphthous or shallow ulcers in the ileocecal area. 
perianal lesions, stricture, and fistula formations are more frequent in $\mathrm{CD}^{11,22}$ Endoscopically, longitudinal ulcers with a cobblestone appearance are frequently observed in CD, whereas intestinal lesions in intestinal BD tend to appear as few round-shaped, punched-out, deep ulcers with discrete margins, which are more prone to perforate. ${ }^{17}$ Lee et al. ${ }^{23}$ suggested simplified algorithms for the differential diagnosis of the 2 disease entities by investigating their colonoscopic features. Five colonoscopy findings, including a round ulcer shape, focal distribution, fewer than 6 ulcers, absence of aphthous lesions, and cobblestone appearance were independently dominant in intestinal BD, and by sequentially applying 2 variables with ulcer shape followed by distribution, the 2 diseases could be differentiated up to $92 \%$.

Intestinal tuberculosis (ITB) may also be difficult to distinguish from intestinal $\mathrm{BD}$ and $\mathrm{CD}$ because of its overlap in endoscopic appearance and prevalent geographic regions. According to a study by Lee et al., ${ }^{24}$ involvement of fewer than 4 segments, patulous ileocecal valves, transverse ulcers, and scars or pseudopolyps were observed commonly in ITB, and anorectal lesions, longitudinal ulcers, aphthous ulcers, and cobblestone appearance were seen frequently in CD. Using these colonoscopic parameters, ITB can be diagnosed correctly in $87.5 \%$ of the patients.

\section{Novel Diagnostic Criteria for Intestinal BD}

According to the Japanese consensus-based practice guidelines for the diagnosis of intestinal BD using a modified Delphi approach, patients should have both confirmed BD and intestinal lesions without other gastrointestinal diseases to enable a definite diagnosis of intestinal BD. ${ }^{21}$ Some patients with typical intestinal ulcers do not meet the diagnostic criteria of $\mathrm{BD}$, and systemic manifestations may appear sequentially after several months or years after initial intestinal ulcerations in others. ${ }^{17,25,26}$ One of the major concerns of the Japanese diagnostic criteria includes intestinal involve- ments in patients without clinical findings of BD. Lee et al. ${ }^{17}$ reported that the clinical and colonoscopic characteristics of patients with intestinal involvement who lack the systemic manifestations of BD were in accordance with the characteristics of those who fulfill the criteria. Shin et al. ${ }^{26}$ also showed that patients who have typical ulcerations without systemic BD symptoms tended to satisfy the BD criteria throughout the disease course. Another concern is that the characteristics of intestinal lesions are difficult to define. Simple intestinal ulcers are often considered as a variation of the same disease spectrum of intestinal BD because intestinal lesions initially presenting as simple ulcers could be eventually diagnosed as intestinal BD over time. ${ }^{27,28}$ Therefore, to date, clinicians are apt to diagnose and manage intestinal BD even in patients who do not fully satisfy the systemic manifestation criteria for $\mathrm{BD}$ at the time of colonoscopy.

Owing to these unmet needs, the Korean IBD Study Group recently developed novel diagnostic criteria for intestinal $\mathrm{BD}^{29}$ These diagnostic criteria were developed on the basis of the colonoscopic findings and extraintestinal systemic manifestations using a modified Delphi method. The extraintestinal manifestations were classified in accordance with the diagnostic criteria by the BD Research Committee of Japan ${ }^{16}$ that can reflect a temporal change better than the International Study Group for BD criteria during followup. The newly developed criteria categorized patients into 4 groups: definite, probable, suspected, and nondiagnostic (Fig. 2) and suggested a management strategy according to each category. Patients with typical intestinal ulcerations and extraintestinal symptoms who met the Japanese criteria were categorized as the "definite" intestinal BD group and managed for intestinal BD. Patients with typical intestinal ulcerations with only typical oral symptoms or atypical intestinal ulcerations and extraintestinal symptoms who met the Japanese criteria were categorized as the "probable" group. Patients with typical intestinal ulcerations without any extraintestinal symptoms or atypical intestinal ulcerations with

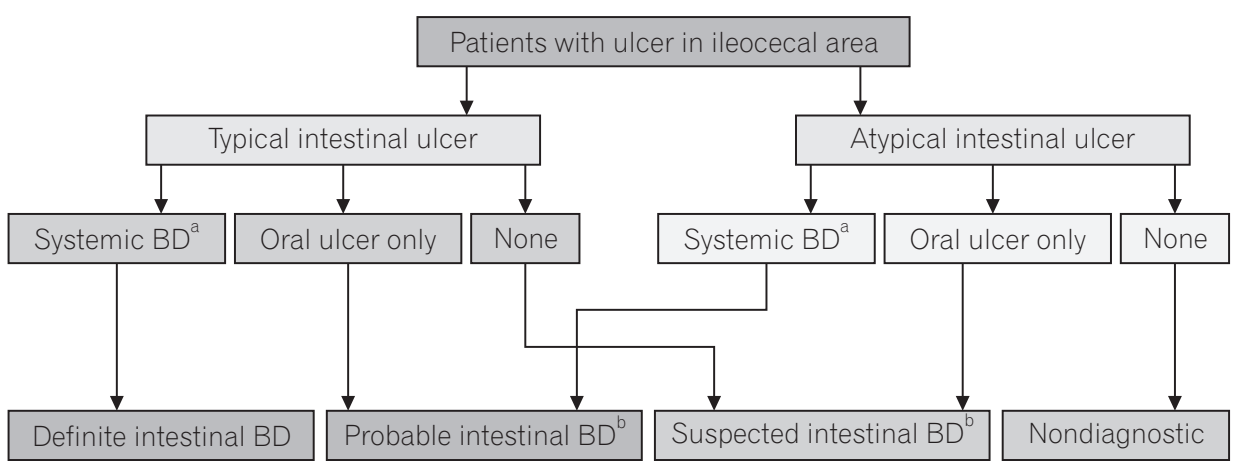

Fig. 2. Algorithm for the diagnosis of intestinal Behçet's disease (BD) based on the type of ileocolonic ulcerations and clinical manifestations. ${ }^{\text {TT}}$ The subtypes of systemic BD (complete, incomplete, and suspected) were classified in accordance with the diagnostic criteria of the Research Committee of Japan; ${ }^{b} \mathrm{~A}$ close follow-up is necessary. Adapted from Cheon $\mathrm{JH}$, et al. ${ }^{29}$ 
only typical oral symptoms were categorized as the "suspected" group. Patients in the probable or suspected group needed to be followed up closely because a substantial number of patients are eventually diagnosed with intestinal BD. The "nondiagnostic" group included patients who had only atypical intestinal ulcerations without any extraintestinal manifestations. These newly developed algorithms for the diagnosis of intestinal BD might be useful, especially in patients with intestinal ulcerations in the ileocecal area who do not fully satisfy the diagnostic criteria of systemic BD.

\section{DISEASE ACTIVITY MONITORING OF INTESTINAL BD}

Intestinal BD showed a heterogeneous range of clinical courses over time. In a retrospective study by Jung et al. ${ }^{30}$ analyzing the clinical course of patients with intestinal BD who were regularly followed up for at least 5 years, majority of the patients $(71.6 \%)$ had a mild clinical activity, whereas the remaining patients (28.4\%) had multiple relapses or chronic symptoms. In addition, intestinal BD often requires surgery because of complications, including massive bleeding, fistula formation, and perforation. ${ }^{7,9}$ The cumulative rates of surgery were reported to be $31.6 \%$ at 5 years and $44.4 \%$ at 10 years after diagnosis, which were comparable to those of CD. ${ }^{11}$ Even after surgery, the cumulative clinical recurrence rates were shown to be $21.1 \%$ at 1 year and $51.1 \%$ at 5 years. ${ }^{11}$ Considering its unpredictable disease flare-ups and poor overall prognosis, the objective assessment of its disease activity is indispensable in determining the best therapeutic strategy and in assessing the treatment response.

\section{Endoscopy}

Endoscopy plays an essential role in the measurement of the disease extent and severity of IBD. ${ }^{31}$ Kim et al. ${ }^{18}$ analyzed the clinical outcomes of patients with intestinal BD according to the colonoscopy findings that volcano-type ulcerations were prone to show less favorable responses to medications, more frequent surgical intervention, and recurrence than the aphthous and geographic ulcerations. Lee et al. ${ }^{32}$ also suggested that more than 2 ulcers and volcano-shaped ulcers were associated with higher disease activities.

As mucosal healing has emerged as a treatment target in IBD, the need for endoscopy continues to increase. ${ }^{33,34}$ In agreement with previous studies in IBD, ${ }^{35,36}$ Yim et al. ${ }^{37}$ reported that mucosal healing was an independent factor for favorable clinical outcomes with a lower recurrence rate in patients with intestinal BD. The presence of active ulcerations was associated with a higher risk of disease relapse even in clinical remission status, which might implicate the role of mucosal healing as a therapeutic target in the management of intestinal BD.

In addition, endoscopy is considered the gold standard in the diagnosis of postoperative recurrence and prediction of clinical outcomes in IBD. The postoperative Crohn's endoscopic recurrence study revealed that early colonoscopy and step-up treatment are better than conventional drug therapy alone for the prevention of postoperative CD recurrence. ${ }^{38}$ Recently, our group developed an endoscopic scoring system predicting clinical relapse after surgery in patients with intestinal BD using colonoscopic findings, including ulcer size and numbers. Multiple and $>20 \mathrm{~mm}$-sized ulcers were related to clinical relapse after surgery in patients with intestinal BD (data unpublished). However, while widely accepted to represent the state of bowel inflammation, endoscopy still has limitations of cost, inconvenience, and invasiveness.

\section{Biomarkers}

To complement these drawbacks, various biomarkers have been studied to estimate the severity of inflammation and monitor the disease activities. Among laboratory markers, ESR and CRP are the most studied and considered the most representative. ${ }^{39,40}$ ESR and CRP levels were well correlated with the disease activity of systemic $\mathrm{BD},{ }^{41,42}$ and a higher CRP level was proven to be a predictor of relapse in patients with intestinal BD with 5-aminosalicylic acid/sulfasalazine treatment. ${ }^{43}$ In addition, cumulative recurrence rates and reoperation rates were higher in surgically treated patients with higher CRP levels than in those with lower CRP. ${ }^{44}$

Choi et al. ${ }^{45}$ found that the positive rate of anti-Saccharomyces cerevisiae antibodies was $44.3 \%$ in patients with intestinal $\mathrm{BD}$; anti-S. cerevisiae antibody positivity was significantly associated with increased surgical rates. In addition, Shin et al. ${ }^{46}$ reported that the prevalence of anti- $\alpha$-enolase antibody was $67.5 \%$ in patients with intestinal $\mathrm{BD}$, and a cumulative steroid use was higher in patients with a positive anti- $\alpha$-enolase antibody than in those with a negative antibody. Recently, serum soluble triggering receptor expressed on myeloid cells-1, which is known to play a role in the inflammatory response by stimulating the productions of proinflammatory cytokines, ${ }^{47}$ has been shown to increase in patients with intestinal BD; further, its levels showed a higher correlation with the disease activity of intestinal BD than CRP and ESR levels. ${ }^{48}$ In addition, a recent study found that levels of IL-12B 
involved in Th17 differentiation were significantly correlated with clinical and endoscopic activities of intestinal BD implicating its potential role as a biomarker. ${ }^{49}$ On the other hand, procalcitonin was able to differentiate infection from active inflammation in IBD in that it was not affected by the disease activity of intestinal BD but by a concomitant infection. ${ }^{50}$

Compared to serologic biomarkers, fecal biomarkers, such as calprotectin and lactoferrin, have the advantage in terms

Table 1. Disease Activity Index for Intestinal Behçet's Disease

\begin{tabular}{|c|c|}
\hline Item & Score \\
\hline \multicolumn{2}{|l|}{ General well-being for 1 week } \\
\hline Well & 0 \\
\hline Fair & 10 \\
\hline Poor & 20 \\
\hline Very poor & 30 \\
\hline Terrible & 40 \\
\hline \multicolumn{2}{|l|}{ Fever $\left({ }^{\circ} \mathrm{C}\right)$} \\
\hline$<38$ & 0 \\
\hline$\geq 38$ & 10 \\
\hline Extraintestinal manifestations ${ }^{a}$ & 5 Per item \\
\hline \multicolumn{2}{|l|}{ Abdominal pain in 1 week } \\
\hline None & 0 \\
\hline Mild & 20 \\
\hline Moderate & 40 \\
\hline Severe & 80 \\
\hline \multicolumn{2}{|l|}{ Abdominal mass } \\
\hline None & 0 \\
\hline Palpable mass & 10 \\
\hline \multicolumn{2}{|l|}{ Abdominal tenderness } \\
\hline None & 0 \\
\hline Mildly tender & 10 \\
\hline Moderately or severely tender & 20 \\
\hline Intestinal complications ${ }^{\mathrm{b}}$ & 10 Per item \\
\hline \multicolumn{2}{|l|}{ No. of liquid stools in 1 week } \\
\hline 0 & 0 \\
\hline $1-7$ & 10 \\
\hline $8-21$ & 20 \\
\hline $22-35$ & 30 \\
\hline$\geq 36$ & 40 \\
\hline
\end{tabular}

${ }^{2}$ Score 5 for oral ulcer, genital ulcer, eye lesion, skin lesion, or arthralgia; score 15 for vascular involvement or CNS involvement.

${ }^{b}$ Fistula, perforation, abscess, or intestinal obstruction. Adapted from Cheon JH, et al. Inflamm Bowel Dis 2011;17:605-613. ${ }^{53}$ of increased specificity for intestinal inflammation. ${ }^{40,51}$ Kim et al. ${ }^{52}$ suggested that similar to IBD, levels of fecal calprotectin were significantly higher in patients with typical ulcerations than in those with atypical ulcerations, and the absolute changes in fecal calprotectin and disease activity index for intestinal BD (DAIBD) showed a significant correlation during 3 months of treatment. However, to date, no single biomarker has been proven to be ideal in assessing the disease activity of intestinal IBD.

\section{Disease Activity Index for Intestinal BD}

To date, there is no specific tool to assess the disease activity of intestinal BD; therefore, the measurement of its disease activity depends heavily on expert opinions or the use of other IBD indices, such as the CDAI. ${ }^{21}$ The Korean IBD Study Group accordingly developed a novel tool to measure the disease activity of intestinal $\mathrm{BD} .^{53}$ As a single clinical or laboratory parameter could not consistently reflect the level of intestinal inflammation, a multi-item measurement similar to $\mathrm{CDAI}^{54}$ and pediatric ulcerative colitis activity index ${ }^{55}$ was needed to evaluate the disease activity of intestinal BD. DAIBD consists of 8 items as follows: general well-being, fever, extraintestinal manifestations, abdominal pain, abdominal mass, abdominal tenderness, intestinal complication, and number of liquid stools (Table 1), ranging from a score of 0 to 325. Depending on the DAIBD score, the disease severity was divided into 4 groups (Table 2): quiescent $(\leq 19)$, mild (20-39), moderate (40-74), and severe ( $\geq 75)$ diseases. DAIBD showed a better performance than CDAI in terms of good correlation with the physician's global assessment. DAIBD has also advantages over CDAI as it is a relatively simple 8-point index and is easily applied in the outpatient clinic. However, the recent study failed to show the correlation between DAIBD and endoscopic severity, similar to CD. ${ }^{32,56}$ Meanwhile, a multinational web-based survey reported that even in Asian countries, physicians adopted dif-

Table 2. Cutoff Scores of the Disease Activity Index for Intestinal Behçet's Disease

\begin{tabular}{cc}
\hline Disease activity & DAIBD score \\
\hline Remission & $\leq 19$ \\
Mild & $20-39$ \\
Moderate & $40-74$ \\
Severe & $\geq 75$ \\
\hline
\end{tabular}

DAIBD, disease activity index for intestinal Behçet's disease.

Adapted from Cheon JH, et al. Inflamm Bowel Dis 2011;17:605-613. ${ }^{53}$ 
ferent scoring systems to assess the disease activity of IBD. ${ }^{57}$ Further studies are mandatory to validate the usefulness of DAIBD in various populations and predict the clinical outcomes more precisely by considering both endoscopic factors and clinical activity indices.

\section{CONCLUSIONS}

Gastrointestinal involvement is one of the major causes of morbidity and mortality in BD. However, clinical data are limited because of the scarcity of intestinal BD cases, and to date, there has been no standardized diagnostic tool or pathognomonic finding for intestinal BD. Recently developed novel diagnostic criteria and a disease activity index might help physicians make a correct diagnosis and decide an appropriate treatment by assessing the disease activity of intestinal BD. Further international validation for these guidelines is needed, and biomarkers specific for intestinal $\mathrm{BD}$ in the diagnosis, disease activity assessment, and clinical outcome prediction should be investigated in the future.

\section{REFERENCES}

1. O'Duffy JD. Behçet's disease. Curr Opin Rheumatol 1994;6:39-43.

2. Kaklamani VG, Vaiopoulos G, Kaklamanis PG. Behçet's disease. Semin Arthritis Rheum 1998;27:197-217.

3. Sakane T, Takeno M, Suzuki N, Inaba G. Behçet's disease. N Engl J Med 1999;341:1284-1291.

4. Baba S, Maruta M, Ando K, Teramoto T, Endo I. Intestinal Behçet's disease: report of five cases. Dis Colon Rectum 1976;19:428-440.

5. Kasahara Y, Tanaka S, Nishino M, Umemura H, Shiraha S, Kuyama T. Intestinal involvement in Behçet's disease: review of 136 surgical cases in the Japanese literature. Dis Colon Rectum 1981;24:103-106.

6. Bayraktar Y, Ozaslan E, Van Thiel DH. Gastrointestinal manifestations of Behcet's disease. J Clin Gastroenterol 2000;30:144-154.

7. Grigg EL, Kane S, Katz S. Mimicry and deception in inflammatory bowel disease and intestinal Behçet disease. Gastroenterol Hepatol (N Y) 2012;8:103-112.

8. Kim DH, Cheon JH. Intestinal Behçet's disease: a true inflammatory bowel disease or merely an intestinal complication of systemic vasculitis? Yonsei Med J 2016;57:22-32.

9. Yang SK. Intestinal Behcet's disease. Intest Res 2005;3:1-10.

10. Kim SU, Cheon JH, Lim JS, et al. Massive gastrointestinal bleeding due to aneurysmal rupture of ileo-colic artery in a patient with Behcet's disease. Korean J Gastroenterol 2007;49:400-404.
11. Jung YS, Cheon JH, Park SJ, Hong SP, Kim TI, Kim WH. Longterm clinical outcomes of Crohn's disease and intestinal Behcet's disease. Inflamm Bowel Dis 2013;19:99-105.

12. Lakhanpal S, Tani K, Lie JT, Katoh K, Ishigatsubo Y, Ohokubo T. Pathologic features of Behçet's syndrome: a review of Japanese autopsy registry data. Hum Pathol 1985;16:790-795.

13. Cheon JH, Shin SJ, Kim SW, et al. Diagnosis of intestinal Behçet's disease. Korean J Gastroenterol 2009;53:187-193.

14. Cheon JH, Kim WH. An update on the diagnosis, treatment, and prognosis of intestinal Behçet's disease. Curr Opin Rheumatol 2015;27:24-31.

15. Criteria for diagnosis of Behçet's disease. International Study Group for Behçet's Disease. Lancet 1990;335:1078-1080.

16. Mizushima Y, Inaba G, Mimura Y, Ono S. Diagnostic criteria for Behçet's disease in 1987, and guidelines for treating Behçet's disease. Saishin Igaku 1988;43:382-391.

17. Lee CR, Kim WH, Cho YS, et al. Colonoscopic findings in intestinal Behçet's disease. Inflamm Bowel Dis 2001;7:243-249.

18. Kim JS, Lim SH, Choi IJ, et al. Prediction of the clinical course of Behçet's colitis according to macroscopic classification by colonoscopy. Endoscopy 2000;32:635-640.

19. Shepherd NA. Pathological mimics of chronic inflammatory bowel disease. J Clin Pathol 1991;44:726-733.

20. Ebert EC. Gastrointestinal manifestations of Behçet's disease. Dig Dis Sci 2009;54:201-207.

21. Kobayashi K, Ueno F, Bito S, et al. Development of consensus statements for the diagnosis and management of intestinal Behçet's disease using a modified Delphi approach. J Gastroenterol 2007;42:737-745.

22. Cheon JH, Celik AF, Kim WH. Behçet's disease: gastrointestinal involvement. In: Yazici Y, Yazici H, eds. Behçet's syndrome. New York: Springer, 2010:165-188.

23. Lee SK, Kim BK, Kim TI, Kim WH. Differential diagnosis of intestinal Behçet's disease and Crohn's disease by colonoscopic findings. Endoscopy 2009;41:9-16.

24. Lee YJ, Yang SK, Byeon JS, et al. Analysis of colonoscopic findings in the differential diagnosis between intestinal tuberculosis and Crohn's disease. Endoscopy 2006;38:592-597.

25. O'Duffy JD. Vasculitis in Behçet's disease. Rheum Dis Clin North Am 1990;16:423-431.

26. Shin SJ, Lee SK, Kim TI, et al. Chronological changes in the systemic manifestations of intestinal Behcet's disease and their significance in diagnosis. Int J Colorectal Dis 2010;25:1371-1376.

27. Muto T. Historical review of so called "simple ulcer" of the intestine. Stomach Intestine 1979;14:739-748.

28. Jung HC, Rhee PL, Song IS, Choi KW, Kim CY. Temporal changes in the clinical type or diagnosis of Behçet's colitis in patients with aphthoid or punched-out colonic ulcerations. J Korean Med Sci 1991;6:313-318. 
29. Cheon JH, Kim ES, Shin SJ, et al. Development and validation of novel diagnostic criteria for intestinal Behçet's disease in Korean patients with ileocolonic ulcers. Am J Gastroenterol 2009;104:2492-2499.

30. Jung YS, Cheon JH, Park SJ, Hong SP, Kim TI, Kim WH. Clinical course of intestinal Behcet's disease during the first five years. Dig Dis Sci 2013;58:496-503.

31. Cheon JH, Kim WH. Recent advances of endoscopy in inflammatory bowel diseases. Gut Liver 2007;1:118-125.

32. Lee HJ, Kim YN, Jang HW, et al. Correlations between endoscopic and clinical disease activity indices in intestinal Behcet's disease. World J Gastroenterol 2012;18:5771-5778.

33. Rutgeerts P, Vermeire S, Van Assche G. Mucosal healing in inflammatory bowel disease: impossible ideal or therapeutic target? Gut 2007;56:453-455.

34. Frøslie KF, Jahnsen J, Moum BA, Vatn MH; IBSEN Group. Mucosal healing in inflammatory bowel disease: results from a Norwegian population-based cohort. Gastroenterology 2007;133:412-422.

35. Rutgeerts P, Sandborn WJ, Feagan BG, et al. Infliximab for induction and maintenance therapy for ulcerative colitis. N Engl J Med 2005;353:2462-2476.

36. Schnitzler F, Fidder H, Ferrante M, et al. Mucosal healing predicts long-term outcome of maintenance therapy with infliximab in Crohn's disease. Inflamm Bowel Dis 2009;15:1295-1301.

37. Yim SM, Kim DH, Lee HJ, et al. Mucosal healing predicts the long-term prognosis of intestinal Behçet's disease. Dig Dis Sci 2014;59:2529-2535.

38. De Cruz P, Kamm MA, Hamilton AL, et al. Crohn's disease management after intestinal resection: a randomised trial. Lancet 2015;385:1406-1417.

39. Vermeire S, Van Assche G, Rutgeerts P. Laboratory markers in IBD: useful, magic, or unnecessary toys? Gut 2006;55:426-431.

40. Sands BE. Biomarkers of inflammation in inflammatory bowel disease. Gastroenterology 2015;149:1275-1285.e2.

41. Müftüoğlu AU, Yazici H, Yurdakul S, et al. Behçet's disease: relation of serum C-reactive protein and erythrocyte sedimentation rates to disease activity. Int J Dermatol 1986;25:235-239.

42. Coskun B, Saral Y, Gödekmerdan A, Erden I, Coskun N. Activation markers in Behçet's disease. Skinmed 2005;4:282-286.

43. Jung YS, Hong SP, Kim TI, Kim WH, Cheon JH. Long-term clinical outcomes and factors predictive of relapse after 5-aminosalicylate or sulfasalazine therapy in patients with intestinal Behcet disease. J Clin Gastroenterol 2012;46:e38-e45.

44. Jung YS, Yoon JY, Lee JH, et al. Prognostic factors and long-term clinical outcomes for surgical patients with intestinal Behcet's disease. Inflamm Bowel Dis 2011;17:1594-1602.
45. Choi CH, Kim TI, Kim BC, et al. Anti-Saccharomyces cerevisiae antibody in intestinal Behçet's disease patients: relation to clinical course. Dis Colon Rectum 2006;49:1849-1859.

46. Shin SJ, Kim BC, Kim TI, Lee SK, Lee KH, Kim WH. Anti-alphaenolase antibody as a serologic marker and its correlation with disease severity in intestinal Behçet's disease. Dig Dis Sci 2011;56:812-818.

47. Bouchon A, Dietrich J, Colonna M. Cutting edge: inflammatory responses can be triggered by TREM-1, a novel receptor expressed on neutrophils and monocytes. J Immunol 2000;164:4991-4995.

48. Jung YS, Kim SW, Yoon JY, et al. Expression of a soluble triggering receptor expressed on myeloid cells-1 (sTREM-1) correlates with clinical disease activity in intestinal Behcet's disease. Inflamm Bowel Dis 2011;17:2130-2137.

49. Lee HW, Chung SH, Moon CM, et al. The correlation of serum IL-12B expression with disease activity in patients with inflammatory bowel disease. Medicine (Baltimore) 2016;95:e3772. doi: 10.1097/MD.0000000000003772.

50. Chung SH, Lee HW, Kim SW, et al. Usefulness of measuring serum procalcitonin levels in patients with inflammatory bowel disease. Gut Liver 2016;10:574-580.

51. Wei SC. Could fecal calprotectin enter mainstream use for diagnosing and monitoring inflammatory bowel disease? Intest Res 2016;14:293-294.

52. Kim DH, Park Y, Kim B, et al. Fecal calprotectin as a non-invasive biomarker for intestinal involvement of Behçet's disease. J Gastroenterol Hepatol 2017;32:595-601.

53. Cheon JH, Han DS, Park JY, et al. Development, validation, and responsiveness of a novel disease activity index for intestinal Behçet's disease. Inflamm Bowel Dis 2011;17:605-613.

54. Best WR, Becktel JM, Singleton JW, Kern F Jr. Development of a Crohn's disease activity index: National Cooperative Crohn's Disease Study. Gastroenterology 1976;70:439-444.

55. Turner D, Otley AR, Mack D, et al. Development, validation, and evaluation of a pediatric ulcerative colitis activity index: a prospective multicenter study. Gastroenterology 2007;133:423-432.

56. Cellier C, Sahmoud T, Froguel E, et al. Correlations between clinical activity, endoscopic severity, and biological parameters in colonic or ileocolonic Crohn's disease: a prospective multicentre study of 121 cases. The Groupe d'Etudes Thérapeutiques des Affections Inflammatoires Digestives. Gut 1994;35:231-235.

57. Kim ES, Chen M, Lee J, Lee CK, Kim YS. Diagnosis of inflammatory bowel disease in Asia: the results of a multinational webbased survey in the 2(nd) Asian Organization for Crohn's and Colitis (AOCC) meeting in Seoul. Intest Res 2016;14:224-230. 